\title{
Commissioning and cross-comparison of four scanning water tanks
}

\author{
Daniel Saenz ${ }^{1}$, Joseph Roring1, Wilbert Cruz ${ }^{1}$, Vikren Sarkar², \\ Niko Papanikolaou' ${ }^{1}$, Sotirios Stathakis ${ }^{1}$
}

${ }_{1}^{1}$ Department of Radiation Oncology, University of Texas Health Science Center - San Antonio, San Antonio, Texas, USA 2Department of Radiation Oncology, University of Utah, Salt Lake City, Utah, USA

Received October 13, 2015; Revised November 24, 2015; Accepted December 01, 2015; Published Online December 04, 2015

\section{Technical Report}

\begin{abstract}
Purpose: Water scanning systems are commonly used for data collection to characterize dosimetric properties of photon and electron beams, and the commissioning of such systems has been previously described. The aim in this study, however, was to investigate tank-specific dependencies as well as conduct a dosimetric comparison between four distinct water scanning systems. Methods: Four water scanning systems were studied including the PTW MP3-M Phantom Tank, the Standard Imaging DoseView 3D, the IBA Blue Phantom, and the Sun Nuclear 3D Scanner. Mechanical accuracy and reproducibility was investigated by driving the chamber holder to nominal positions relative to a zero point and using a leveled caliper with $30 \mathrm{~cm}$ range to measure the actual position. Dosimetric measurements were also performed not only to compare percent-depth-dose (PDD) curves and profiles between tanks but also to assess dependencies such as directionality, scanning speed, and reproducibility for each tank individually. A PTW Semiflex 31010 ionization chamber with a sensitive volume of $0.125 \mathrm{cc}$ was used at a Varian Clinac 2300 linear accelerator. Results: Mechanical precision was ensured to within $0.1 \mathrm{~mm}$ with the standard deviation (SD) of reproducibility $<0.1 \mathrm{~mm}$ for measurements made with calipers. Dependencies on scanning direction and speed are presented. $6 \mathrm{MV}$ PDDs between tanks agreed to within $0.6 \%$ relative to an averaged PDD beyond $d_{\max }$ and within $2.5 \%$ in the build-up region. Specifically, the maximum difference was $1.0 \%$ between MP3-M and Blue Phantom at $6.1 \mathrm{~cm}$ depth. Lateral profiles agreed between tanks within $0.5 \%$ in the central $80 \%$ of the field. $6 \mathrm{MeV}$ PDD maximum difference was $1.3 \%$ occurring at the steepest portion, where the $\mathrm{R}_{50}$ was nevertheless within $0.6 \mathrm{~mm}$ across tanks. Setup uncertainties estimated at $\leq 1 \mathrm{~mm}$ are presumed to have contributed some of the difference between water tank data. Conclusion: Modern water scanning systems have achieved high accuracy across vendors, but commissioning tests nevertheless reveal tank-specific dependencies. This study not only ensures confidence in the individual systems but also provides the medical physicist with an understanding of variation in water tank properties between vendors.
\end{abstract}

Keywords: Water Scanning Systems; Beam Commissioning; Radiation Measurements

\section{Introduction}

Water scanning systems have been used for decades to characterize the dose distribution from photon and electron beams in radiotherapy. The quality assurance for water scanning systems was first described by Mellenberg et al. ${ }^{1}$ in 1990 as well as by Purdy ${ }^{2}$ in 1992.
These reports describe the acceptance testing for mechanical components of the water scanning system as well as electrometer and data processing tools. Water scanning systems (water tanks) are used for a wide array of linear accelerator commissioning

Corresponding author: Sotirios Stathakis; Department of Radiation Oncology, University of Texas Health Science Center - San Antonio, San Antonio, Texas, USA.

Cite this article as: Saenz D, Roring J, Cruz W, Sarkar V, Papanikolaou N, Stathakis S. Commissioning and cross-comparison of four scanning water tanks. Int J Cancer Ther Oncol 2016; 4(1):415. D0I: 10.14319/ijcto.41.5 
measurements, including but not limited to percent depth dose (PDD) and in-plane and cross-plane profiles as described in AAPM Task Group \#106. ${ }^{3}$ Through the use of stepper motors, the accuracy and precision of modern water scanning systems is excellent at 0.1 mm. ${ }^{4-6}$ Water scanning systems are used not only for conventional linear accelerators but for special modalities such as CyberKnife ${ }^{7}$ and TomoTherapy ${ }^{8}$.

A recent study by Akino et al. ${ }^{9}$ compared the IBA Blue Phantom, PTW MP3, Standard Imaging DoseView 3D, and Sun Nuclear 3D Scanner to assess the inter-tank variability between dosimetric data. Their results showed sub-percentage agreement between water tanks for profiles and PDD curves. The study concluded in a demonstrable manner that all water tanks studied are capable of acquiring accurate dosimetric beam data. However, this study did not look into dependencies specific to each water tank. More tank-specific data can aid the medical physicist in water tank selection and provide expected capabilities to study during acceptance testing. Moreover, the understanding of factors such as integration time dependence can help in the specification of scanning protocols. Furthermore, the aforementioned study utilized four different ionization chambers in data collection. The use of differing chambers complicates the results, leading the physicist to question if the results would be even more in agreement if the same detector were used. Finally, no electron beam data measurements with different water tanks has been reported to date as far as the authors are aware, which may be even more sensitive to water tank variations given the very sharp dose gradients in electron PDDs. To answer these questions, we studied the four scanning water phantoms for tank specific measurements and cross-tank agreement with one ionization chamber for $6 \mathrm{MV}$ photon PDDs and profiles and $6 \mathrm{MeV}$ electron PDDs.

\section{Methods and Materials}

Tests were conducted on all four water scanning systems across multiple institutions including the DoseView 3D (Standard Imaging, Middleton, WI), MP3-M Phantom Tank (PTW, Freiburg, Germany), Blue Phantom (IBA Dosimetry, Schwarzenbruck, Germany), and 3D Scanner (Sun Nuclear, Melbourne, FL). The first three were studied at institution A with a Varian Clinac 2300 and a PTW Semiflex 31010 ionization chamber with an active volume of $0.125 \mathrm{cc}$. The fourth phantom was studied at institution B with a Varian Clinac iX linear accelerator using a PTW N30013 chamber (0.6 cc). Due to the use of different linear accelerators, the data from the 3D Scanner was excluded from the cross-tank dosimetric comparisons. Water tank specific properties were studied alone for the 3D Scanner.

\subsection{Mechanical tests}

Mechanical tests included mechanical positioning accuracy and positioning repeatability. At institution A, these mechanical checks were performed with a caliper with $30 \mathrm{~cm}$ range. Prior to measurements, the water tank was leveled. In all three water tank dimensions, the detector holder (or arm) was set at a zero location and a measurement from the tank wall or other fixed surface to the zero position was made as a zero point for the calipers. The presence of a curved wall on the 3D scanner made this process not practical. The chamber holder or arm was then moved to various positions (from 1 to $200 \mathrm{~mm}$ ) relative to the origin and measurements were made with the calipers leveled. Repeatability was assessed by returning the chamber holder to the origin and then moving to either 100 or $200 \mathrm{~mm}$ on ten occasions. The use of a caliper was preferable to a plumb bob in this case not only due to the amount of time required for a plumb bob to stabilize its position but due to the increased precision afforded by a caliper relative to the bare eye's assessment of a plumb bob position. Vertical positioning is also better assessed with a caliper as opposed to a plumb bob, which does not have a natural use in the vertical direction. Institution B with a curved wall cylindrical phantom studied mechanical properties instead by fixing graph paper to the bottom of the water tank for lateral and longitudinal measurements and with a ruler fastened to the vertical arm for that dimension.

\subsection{Dosimetric tests}

Scanned data was acquired with a $6 \mathrm{MV}$ photon beam and a $6 \mathrm{MeV}$ electron beam. These energies exhibit the sharpest PDD, therefore testing PDD variations most stringently. PDDs were acquired with a field size at isocenter of $10 \times 10 \mathrm{~cm}^{2}$ with an SSD of $100 \mathrm{~cm} \mathrm{(a} 10 \times$ $10 \mathrm{~cm}^{2}$ cone for $6 \mathrm{MeV}$ ). For profiles, the field size was increased to $20 \times 20 \mathrm{~cm}^{2}$ to better appreciate the wider extent of the beam profile, and scan depth was set at 10 $\mathrm{cm}$. Identical chambers were used for the reference detector. The effective point of measurement was taken into account automatically with the built-in TruFix system for the PTW MP3-M tank but was manually applied with a $0.6 \mathrm{r}_{\text {cav }}$ shift for the other water tanks. Each system's built-in electrometer was used during measurement and the software specific to each phantom for data analysis.

Water tank dosimetric data was obtained in order to understand the dependence of percent-depth-dose (PDD) curves and profiles on parameters such as scanning speed, integration time, directionality, and scanning resolution. For instance, fast scanning speeds can result in dosimetric errors on the order of $5 \%$, necessitating the understanding of scanning speed dependence for various scanning water tanks. ${ }^{10}$ For directionality, recommendations have been made to scan PDD curves from depth towards the surface to minimize water tension effects. ${ }^{11-12}$ These effects were studied explicitly for the various water scanning systems. Table 1 summarizes the tests conducted in this study. Unless otherwise specified, scanning parameters 
used for percent depth dose curves and profiles include $1 \mathrm{~mm}$ scanning resolution, $200 \mathrm{~ms}$ integration time, 32 $\mathrm{mm} / \mathrm{s}$ scanning speed, and a scanning range of $0-30 \mathrm{~cm}$ depth (PDDs) and $125 \%$ of the field size at depth (profiles). By default, PDDs were scanned from depth towards the surface, unless that directional dependence is being tested.

For the testing of scanning speed, PDDs were acquired with detector holder speeds of $15 \mathrm{~mm} / \mathrm{s}, 32 \mathrm{~mm} / \mathrm{s}$, and $50 \mathrm{~mm} / \mathrm{s}$ when possible (for the DoseView 3D and MP3-M). For the 3D scanner, the available continuous scanning speeds of $1 \mathrm{~mm} / \mathrm{s}, 2.5 \mathrm{~mm} / \mathrm{s}, 5 \mathrm{~mm} / \mathrm{s}, 8 \mathrm{~mm} / \mathrm{s}$, $16 \mathrm{~mm} / \mathrm{s}$ were used. With the Blue Phantom, the low (15 $\mathrm{mm} / \mathrm{s})$, medium $(30 \mathrm{~mm} / \mathrm{s})$, and high speed $(50 \mathrm{~mm} / \mathrm{s})$ modes were used, which are virtually the same speeds as the DoseView 3D and MP3-M. The integration time dependence for stepwise operation was tested by setting the integration time at $100 \mathrm{~ms}$ and $400 \mathrm{~ms}$. These scanning speeds were chosen as they were the discrete options available on the Standard Imaging DoseView 3D water scanning tank, but the tests were performed on all applicable tanks. Directional dependence was investigated by scanning from $30 \mathrm{~cm}$ depth to the surface and then in the opposite direction. Static versus dynamic PDD accuracy was acquired by driving the chamber to depths of $1.5,3,5,7,10,15,20$, and $30 \mathrm{~cm}$ using the water tank but making charge measurements with an external PTW UNIDOS webline electrometer. Finally, PDD reproducibility was investigated by conducting a scanning upwards PDD five times. For scanned profiles, data was collected in the left-right and right-left direction as well as in the gun-target and target-gun direction.

For a set of PDD or profile curves with a varied parameter (such as scanning speed), the difference at any point is defined as the maximum value at that point in any of the measured curves minus the minimum value at that same point in any of the measured curves. The maximum difference at any point is assessed across all points and reports the largest absolute difference.

Table 1: Summary of tests performed.

\begin{tabular}{llll}
\hline \hline 1. Mechanical Tests & & 2. Dosimetric Testing \\
\hline a. & Detector Positioning Accuracy & a. & Scanning Speed independence \\
& (1-50 mm displacement) & b. & Integration time independence \\
b. Detector Positioning & c. & Static versus dynamic PDD (point measurements versus scanned PDD) \\
Reproducibility (100 and 200 & d. & PDD Direction of scanning dependence (up versus down) \\
mm displacement) & e. PDD reproducibility \\
& f. & Profile direction of travel independence (left versus right, in versus out) \\
\hline \hline
\end{tabular}

Table 2: Results for mechanical accuracy. For displacements 1-50 mm, the mean and standard deviation (SD) of measurements in all three dimensions are shown. For displacements of 100 and $200 \mathrm{~mm}$, ten measurements were made to assess reproducibility in all three dimensions and the mean and SD are shown.

\begin{tabular}{|c|c|c|c|c|c|c|}
\hline \multirow[b]{3}{*}{$\begin{array}{l}\text { Displacement } \\
(\mathrm{mm})\end{array}$} & \multicolumn{6}{|c|}{ Measured Difference (mm) } \\
\hline & \multicolumn{3}{|c|}{ DoseView 3D } & \multicolumn{3}{|c|}{ MP3-M } \\
\hline & $\begin{array}{l}\text { X (toward } \\
\text { pt left) }\end{array}$ & $\begin{array}{l}\text { Y (toward } \\
\text { gantry) }\end{array}$ & $\begin{array}{l}\mathrm{Z} \text { (towards } \\
\text { floor) }\end{array}$ & $\begin{array}{l}X \text { (toward } \\
\text { pt left) }\end{array}$ & $\begin{array}{l}\text { Y (toward } \\
\text { gantry) }\end{array}$ & $\begin{array}{l}\mathrm{Z} \text { (towards } \\
\text { floor) }\end{array}$ \\
\hline 1 & & $0.0 \pm 0.02$ & & & $0.01 \pm 0.01$ & \\
\hline 5 & & $-0.01 \pm 0.02$ & & & $-0.02 \pm 0.01$ & \\
\hline 10 & & $-0.02 \pm 0.03$ & & & $-0.01 \pm 0.02$ & \\
\hline 20 & & $0.00 \pm 0.02$ & & & $-0.02 \pm 0.01$ & \\
\hline 50 & & $0.02 \pm 0.06$ & & & $0.00 \pm 0.03$ & \\
\hline 100 & $-0.08 \pm 0.03$ & $0.00 \pm 0.02$ & $0.04 \pm 0.03$ & $-0.02 \pm 0.02$ & $-0.03 \pm 0.04$ & $-0.05 \pm 0.02$ \\
\hline \multirow[t]{3}{*}{200} & $0.01 \pm 0.02$ & $0.01 \pm 0.02$ & $0.02 \pm 0.01$ & $-0.10 \pm 0.02$ & $0.00 \pm 0.02$ & $-0.05 \pm 0.01$ \\
\hline & \multicolumn{6}{|c|}{ Measured Difference (mm) } \\
\hline & \multicolumn{3}{|c|}{ Blue Phantom* } & \multicolumn{3}{|c|}{ 3D Scanner** } \\
\hline $\begin{array}{l}\text { Displacement } \\
(\mathrm{mm})\end{array}$ & $\begin{array}{l}\mathrm{X} \text { (toward } \\
\text { pt left) }\end{array}$ & $\begin{array}{l}\mathrm{Y} \text { (toward } \\
\text { gantry) }\end{array}$ & $\begin{array}{l}\mathrm{Z} \text { (towards } \\
\text { floor) }\end{array}$ & $\begin{array}{l}\mathrm{X} \text { (toward } \\
\text { pt left) }\end{array}$ & $\begin{array}{l}\text { Y (toward } \\
\text { gantry) }\end{array}$ & $\begin{array}{l}\mathrm{Z} \text { (towards } \\
\text { floor) }\end{array}$ \\
\hline 1 & & $0.00 \pm 0.02$ & & & $0.00 \pm 0.02$ & \\
\hline 5 & & $0.00 \pm 0.01$ & & & $0.00 \pm 0.01$ & \\
\hline 10 & & $0.02 \pm 0.01$ & & & $0.00 \pm 0.01$ & \\
\hline 20 & & $0.04 \pm 0.01$ & & & $-0.33 \pm 0.01$ & \\
\hline 50 & & $-0.04 \pm 0.04$ & & & $0.00 \pm 0.04$ & \\
\hline 100 & $0.05 \pm 0.04$ & $0.01 \pm 0.03$ & $0.05 \pm 0.02$ & $-0.20 \pm 0.42$ & $-0.10 \pm 0.32$ & $0.00 \pm 0.00$ \\
\hline 200 & $0.06 \pm 0.02$ & $-0.01 \pm 0.02$ & $0.01 \pm 0.01$ & $0.09 \pm 0.30$ & $-0.09 \pm 0.30$ & $0.00 \pm 0.00$ \\
\hline
\end{tabular}




\section{Results}

\subsection{Mechanical tests}

Table 2 outlines the mechanical accuracy tests for all the water tanks. For displacements of 1 through $50 \mathrm{~mm}$, the numbers are mean and standard deviation (SD) across measurements made in all three dimensions. For displacements of 100 and $200 \mathrm{~mm}$, the numbers are mean and SD in each dimension with ten measurements each. The average absolute deviation across all three dimensions and across all displacements was $0.02 \mathrm{~mm}$ for DoseView and MP3-M, $0.1 \mathrm{~mm}$ for the 3D Scanner, and $0.03 \mathrm{~mm}$ for the Blue Phantom. Maximum deviations were $0.09 \mathrm{~mm}, 0.1 \mathrm{~mm}$, and $0.08 \mathrm{~mm}$ for the DoseView, MP3-M, and Blue Phantom, respectively. Maximum recorded deviations were $1 \mathrm{~mm}$ for the 3D Scanner due to the difficulty of the measurement method used at institution B with graph paper attached to the bottom of the tank. The accuracy in reality is expected to be better.

\subsection{Dosimetric tests}

a) Tank-specific dependencies

The first PDD test examined the effect of detector holder scanning speed, whether continuously scanned (Blue Phantom and 3D Scanner) or between measurement points for discrete scans (DoseView 3D and MP3-M). At $\mathrm{d}_{\max }$ and beyond, the maximum PDD difference between high and low speed scanning at any point was $0.40 \%$ for the DoseView 3D tank, $0.38 \%$ for the MP3-M tank, $0.80 \%$ for the Blue Phantom, and $0.33 \%$ for the 3D Scanner. Figure 1 shows the PDD agreement with varied scanning speed for each tank. Note that the difference between PDDs is shown on a secondary axis. When varying the integration time, maximum PDD difference between $400 \mathrm{~ms}$ and $100 \mathrm{~ms}$ integration time PDDs at any point was $0.5 \%$ for the DoseView $3 \mathrm{D}$ and $0.45 \%$ for the MP3-M tank. Figure 2 similarly displays the PDD results with varied integration time.
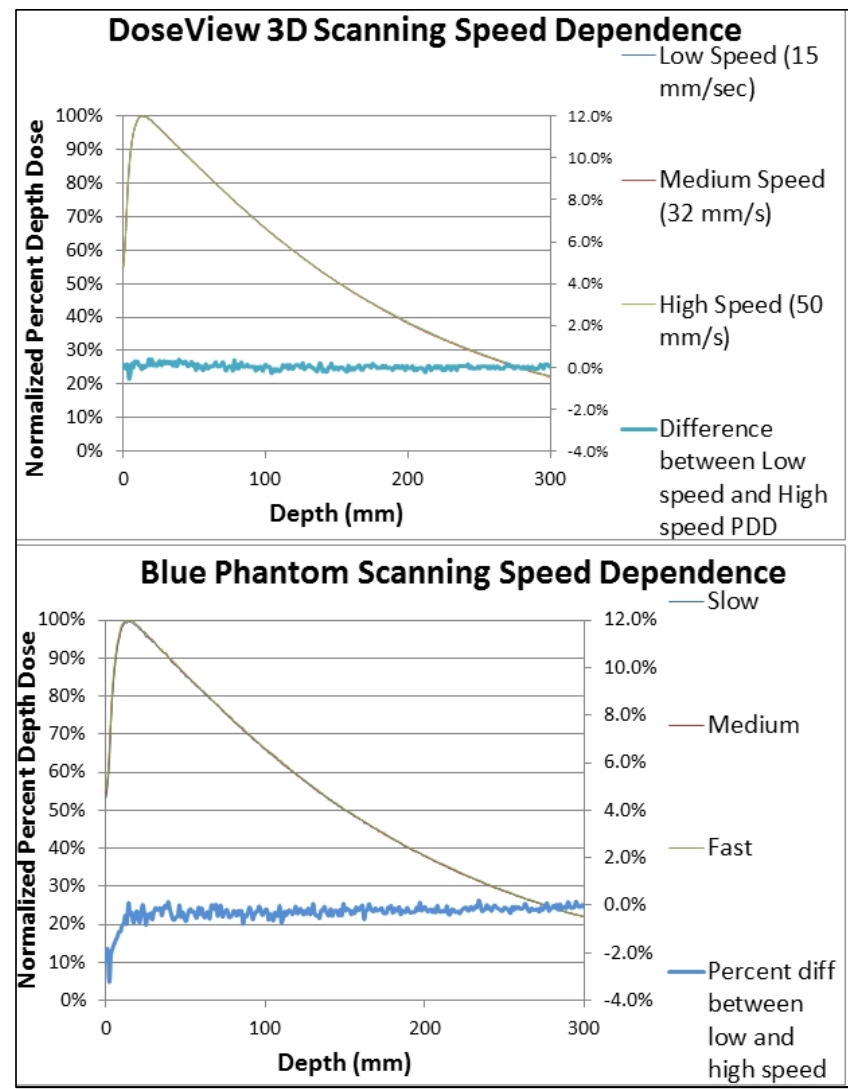

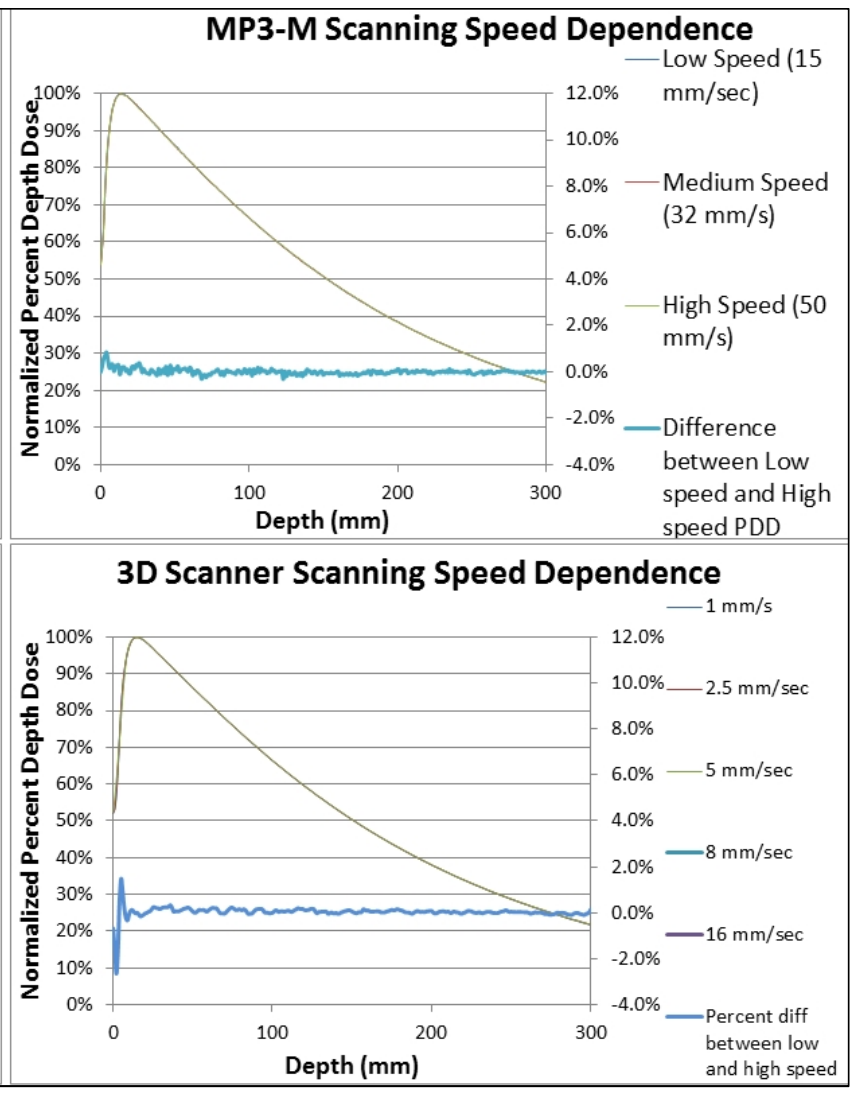

Figure 1: PDD with varied scanning speed for each tank for a 6 MV $10 \times 10 \mathrm{~cm}^{2}$ field in the primary axis. In the secondary axis, the percent difference between the highest and lowest speed scanning is shown. 

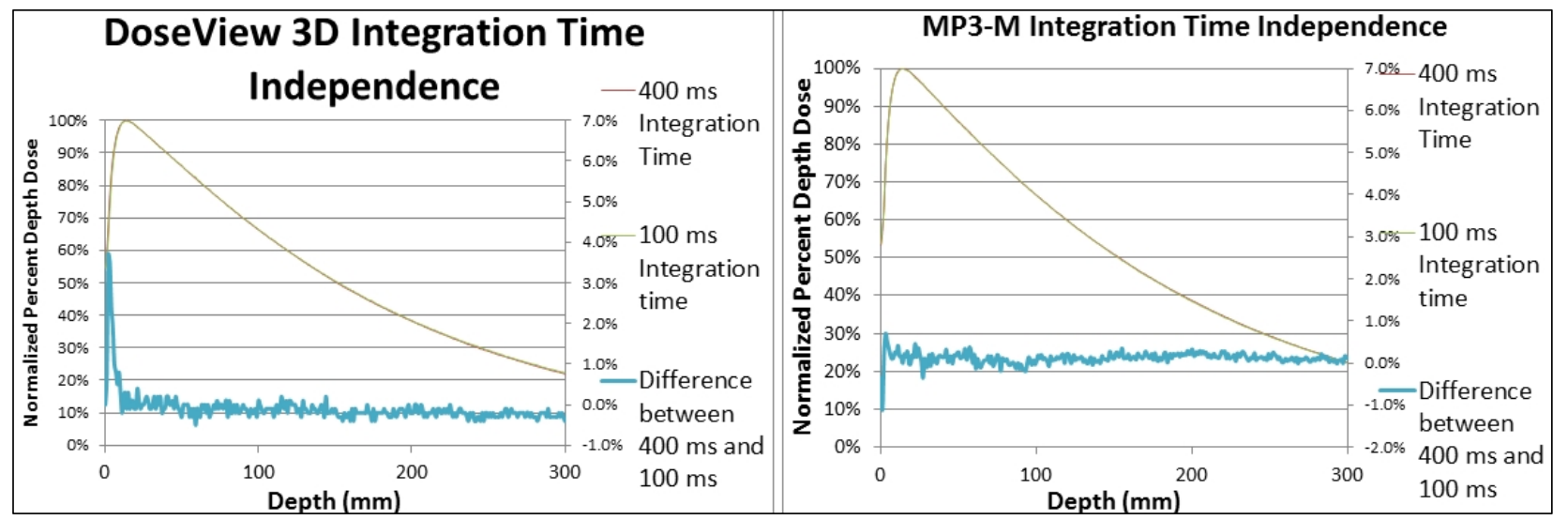

Figure 2: PDD dependence on integration time for those tanks not employing continuous scanning for a $6 \mathrm{MV} 10 \times 10 \mathrm{~cm}^{2}$ field.
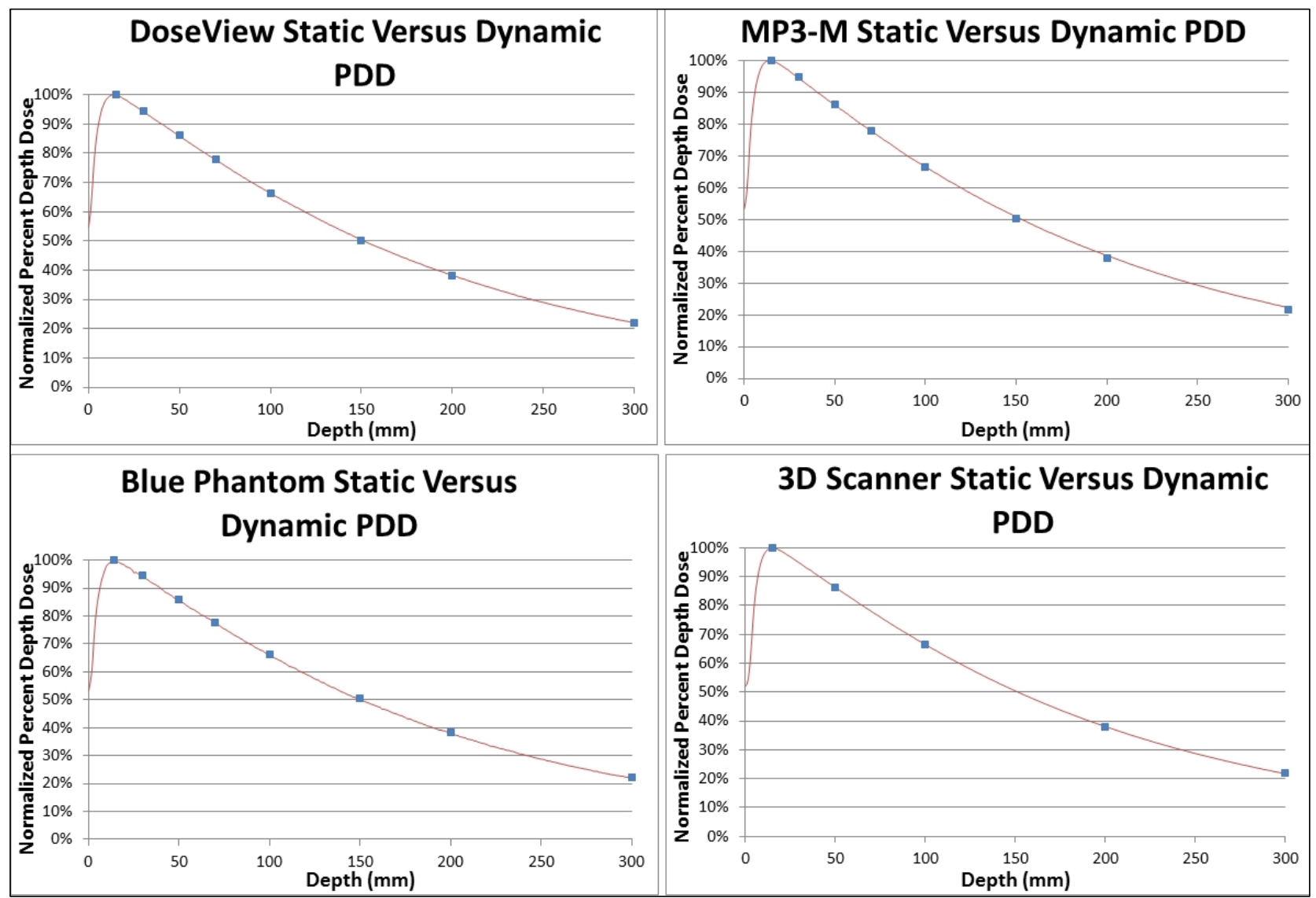

Figure 3: Static versus dynamically acquired PDDs for a $6 \mathrm{MV} 10 \times 10 \mathrm{~cm}^{2}$ field. Static measurements were taken at $1.5 \mathrm{~cm}, 3$ $\mathrm{cm}, 5 \mathrm{~cm}, 7 \mathrm{~cm}, 10 \mathrm{~cm}, 15 \mathrm{~cm}, 20 \mathrm{~cm}$, and $30 \mathrm{~cm}$. Institution B measured at $1.5 \mathrm{~cm}, 5 \mathrm{~cm}, 10 \mathrm{~cm}, 20 \mathrm{~cm}$, and $30 \mathrm{~cm}$.

The static versus dynamic PDD measurements all agreed within $0.3 \%$ for the DoseView 3D, $0.7 \%$ for the MP3-M tank, $0.7 \%$ for the Blue Phantom, and $0.2 \%$ for the 3D Scanner. Figure 3 illustrates the dynamically acquired PDD curve with the static point measurements overlaid.

Directional dependence is shown in Figure $\mathbf{4}$ where all differences are plotted on the same graph. Beyond $d_{\text {max }}$, the maximum difference between scanning up and down was $0.40 \%$ for the DoseView 3D, $0.40 \%$ for the MP3-M, $0.60 \%$ for the Blue Phantom, and $0.53 \%$ for the 3D Scanner. Including the buildup region, there were consistent differences across most of the scanners with the maximum difference of $14.7 \%$ for the DoseView 3D, $14.6 \%$ for the MP3-M, $13.7 \%$ for the Blue Phantom, and $6.6 \%$ for the 3D Scanner. 


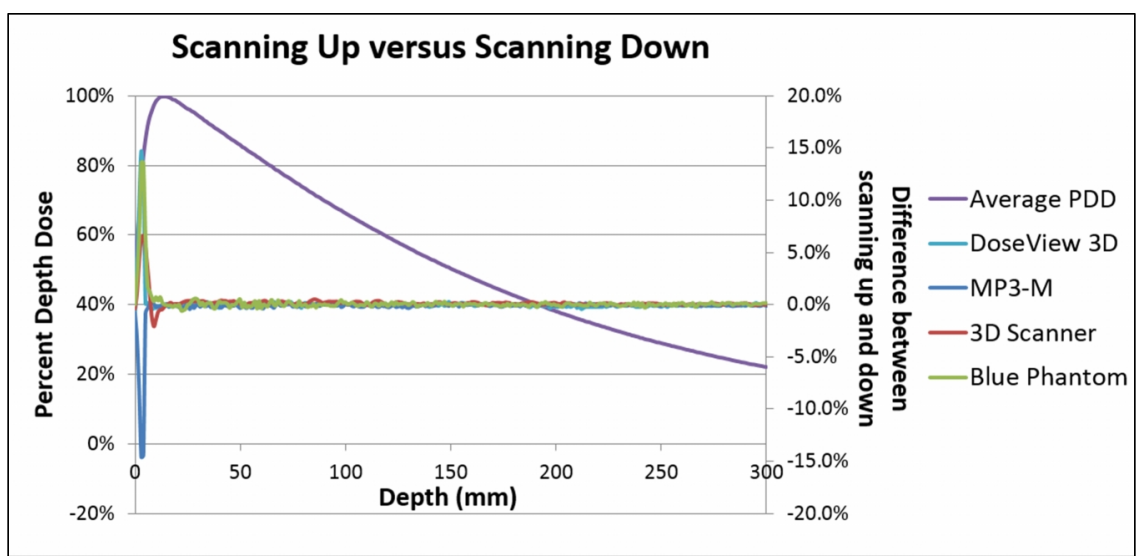

Figure 4: PDD direction of scanning dependence for a $6 \mathrm{MV} 10 \times 10 \mathrm{~cm}^{2}$ field. The difference between scanning up and scanning down is shown for each tank.

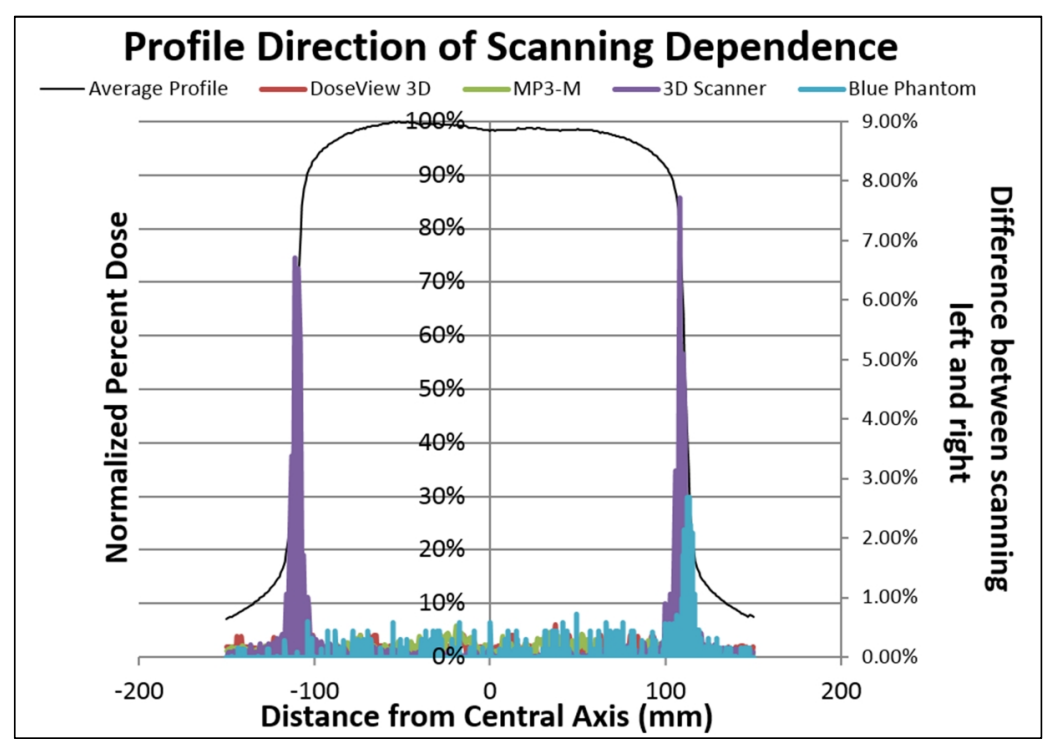

Figure 5: Profile direction of scan dependence for a $6 \mathrm{MV}, 20 \times 20 \mathrm{~cm}^{2}$ field scanned at $10 \mathrm{~cm}$ depth.

For PDD reproducibility, the maximum difference of the five repeated measurements at any depth beyond $\mathrm{d}_{\max }$ was $0.5 \%, 0.6 \%, 0.5 \%$, and $0.9 \%$ for the DoseView 3D, MP3-M, 3D-Scanner, and Blue Phantom, respectively. When including the buildup regions, the maximum difference between measurements climbs to $1.6 \%, 1.2 \%$, $5 \%$, and $1.3 \%$. In terms of standard deviation measured at each point between the five measurements, the maximum SD was $0.21 \%, 0.24 \%, 0.21 \%$, and $0.40 \%$, respectively.

Sub-percentage agreement between profiles scanned left-right versus right-left and gun-target versus target-gun in the central $80 \%$ of the field was obtained for all four scanners $(0.5 \%, 0.5 \%, 0.7 \%, 0.2 \%$ for cross-plane agreement for the DoseView 3D, MP3-M, Blue Phantom, and 3D Scanner respectively). The in-plane numbers were similar at $(0.5 \%, 0.9 \%, 0.7 \%$, $0.2 \%)$. The disagreement in the penumbra regions varied between tanks at $7.7 \%$ for the 3D Scanner (despite the best agreement among tanks in the central
$80 \%$ of the field) and as low as $0.5 \%$ for the MP3-M. The profile differences between scanning left and right in the cross-plane direction for all four tanks are shown in Figure 5.

b) Cross-tank agreement

Figure 6 illustrates the percentage depth-dose agreement for $6 \mathrm{MV}$ photons and $6 \mathrm{MeV}$ electrons between the three tanks with data collected at institution A. For the percent error, the data for each tank was compared to an average PDD, and the maximum difference for any tank relative to the average PDD at any point is plotted. The maximum difference at any depth beyond $\mathrm{d}_{\max }$ was $0.6 \%$. The most variation was present with the Blue Phantom, for which continuous scanning lead to a more unsteady curve. For agreement between DoseView 3D and MP3-M alone, the maximum difference at any depth beyond $\mathrm{d}_{\max }$ was $0.5 \%$. Alternatively, one can look at the worst-case scenario maximum difference between the PDDs at any point. The highest difference was $1.0 \%$ between the MP3-M and 
Blue Phantom at a depth of $6.1 \mathrm{~cm}$. For electrons, the maximum difference at any depth was $1.3 \%$, which occurred in the steep, descending portion of the PDD where the $\mathrm{R}_{50}$ nevertheless agreed within $0.6 \mathrm{~mm}$. In the build-up region and the bremsstrahlung tail, agreement was within $0.5 \%$ and $0.3 \%$ relative to the average PDD, respectively. Finally, when eliminating the Blue Phantom electron PDDs from Figure 6, agreement even along the steep dose gradient is reduced to under $0.5 \%$ compared to over $1 \%$.

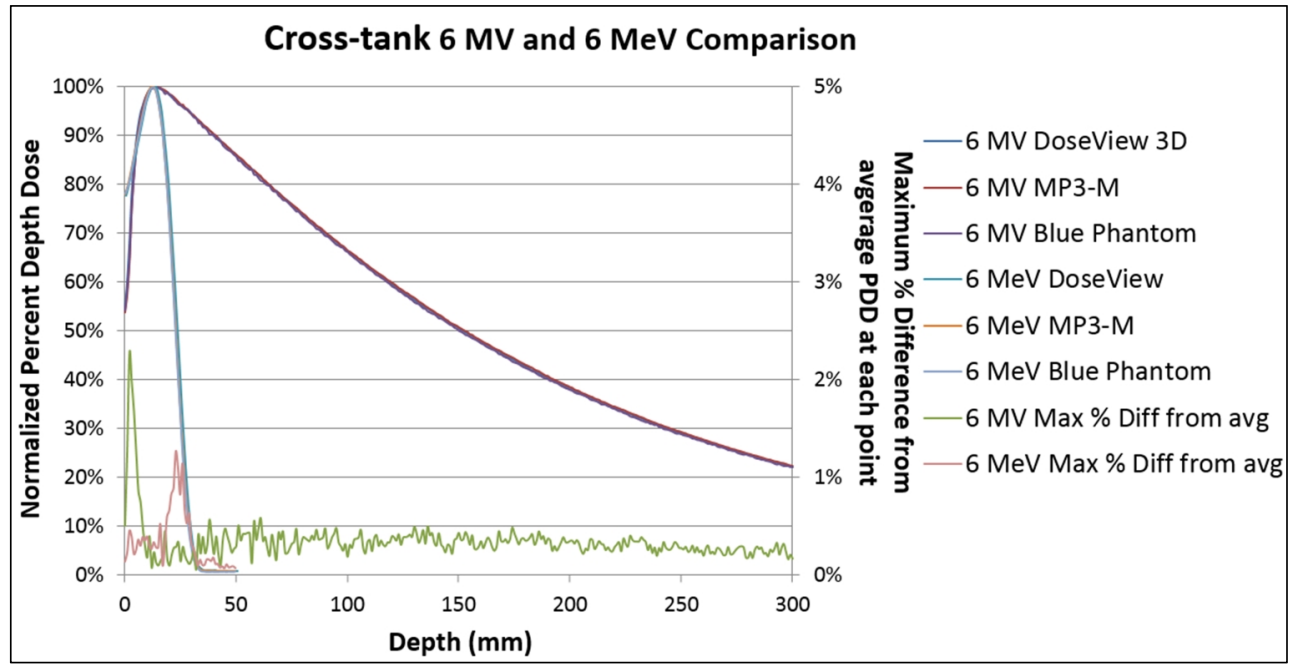

Figure 6: PDD curves acquired for the three water scanning systems at institution A for the 6 MV $10 \times 10 \mathrm{~cm}^{2}$ field and the 6 $\mathrm{MeV}$ beam with a $10 \times 10 \mathrm{~cm}^{2}$ cone.

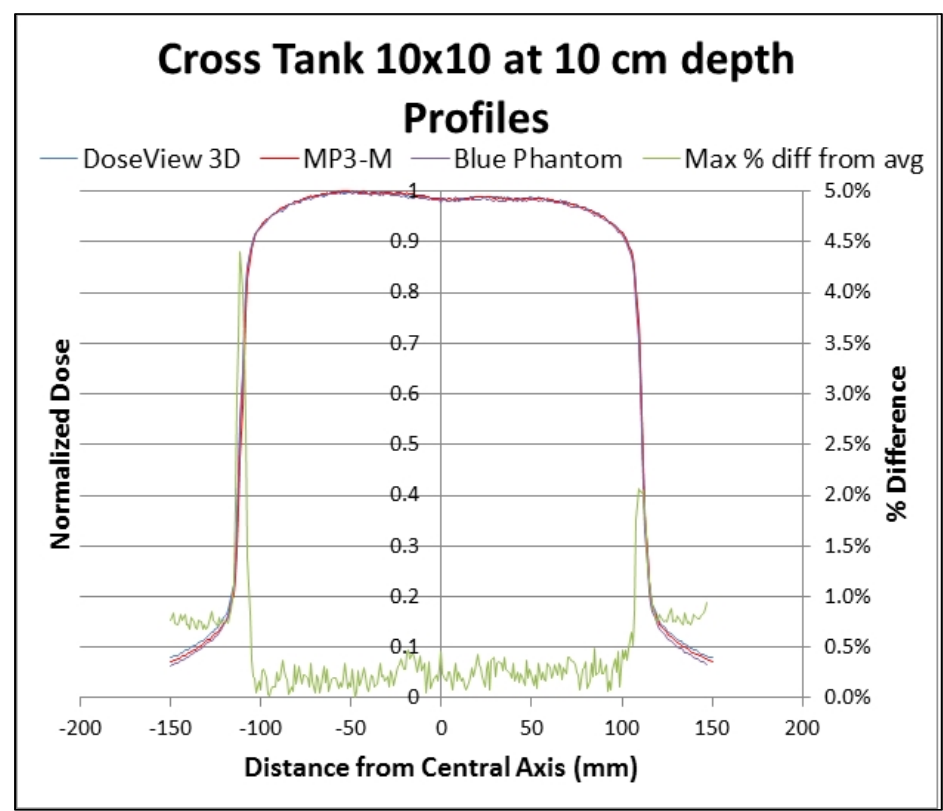

Figure 7: Profiles for a $6 \mathrm{MV}, 20 \times 20 \mathrm{~cm}^{2}$ field scanned at $10 \mathrm{~cm}$ depth acquired with the three tanks at institution A.

Agreement between the profiles collected is shown in Figure 7. Data again is shown for the tanks used at the same institution (DoseView 3D, MP3-M, and Blue Phantom). Here, the maximum difference relative to the average profile within the central $80 \%$ of the field was within $0.5 \%$, with nearly $1 \%$ difference out of field possibly due to variation in electrometer response in low dose regions.

\section{Discussion}

Though 3D water scanning systems have evolved a great deal, the acceptance testing and quality assurance of water tanks remains a critical step to ensure accurate dosimetric data acquisition. Excellent agreement is observed between the four water tanks in this study indicating that no one tank is significantly "better" than another. More important are the tank-specific 
dependencies one needs to understand when setting scanning protocols including speed, direction, or continuous versus discrete stepping. For example, the impacts of continuous versus discrete scanning was observed in the cross-tank PDD comparisons, so particular thought may be given to a system allowing both (3D Scanner). Profile scanning hysteresis was also particularly interesting with the smallest amount in the MP3-M.

The values presented in this study can be compared with the commissioning results obtained by a medical physicist. For instance, one of the first dosimetric tests in the commissioning of a scanning water phantom should be an assessment of scan repeatability. The maximum difference for five scanned PDDs with the specified parameters in this study was sub-percentage. One should therefore expect the maximum difference at any point to be less than $1 \%$ as well (with the specified scanning parameters). Points where the difference is greater than $1 \%$ could be further investigated. Points beyond $d_{\max }$ where the $\mathrm{SD}$ is above $0.5 \%$ may also be suspect. The use of the maximum difference at any point rather than the mean difference allows for a more conservative worst-case scenario criteria. After the scanning repeatability is established, the investigation of other parameter dependencies can be explored.

The alignment of a scanning water phantom with the linear accelerator isocenter includes systematic and random uncertainties. While many of these uncertainties are critical to ensuring dosimetric accuracy for linear accelerator commissioning, some are of little consequence when investigating tank-specific parameter dependences alone (e.g. scanning up versus down). Nevertheless, one cannot expect to align a water phantom so that all points in the phantom agree with machine coordinates by better than $1 \mathrm{~mm}$, introducing a systematic error in measured data. In addition, the radiation/mechanical isocenter agreement may only be within $1 \mathrm{~mm}$. It is crucial to check the radiation center of the beam before commencing beam data acquisition which can partially account for radiation/mechanical isocenter differences as well as chamber positioning errors.

\section{Conclusion}

Modern scanning water tanks have reached a state of high accuracy and precision, mechanically verified here to within $0.1 \mathrm{~mm}$ for the PTW MP3-M, the Standard Imaging DoseView 3D, and the IBA Blue Phantom with a SD of reproducibility of a tenth of a millimeter. Millimeter or better accuracy was demonstrated in the Sun Nuclear 3D Scanner as well. For cross-tank comparisons, $0.6 \%$ agreement for PDD scans beyond $\mathrm{d}_{\max }$ was observed. Lateral profiles were quite comparable between tanks (within $0.5 \%$ in the central field). The profile results were comparable to the Akino et al. ${ }^{9}$ study which presented maximum differences of
$0.8 \%$. The scanning repeatability results beyond $d_{\max }$ in that study (SD $<0.5 \%$ ) were also verified here at $0.4 \%$ or less. Scanning speed dependencies and integration time dependencies were all sub-percentage, but PDD directionality dependencies were nearly $15 \%$ different in the buildup region, supplying experimental evidence of the distinction. Despite the advances in modern scanning water tanks, the percentage errors for the various dependencies are valuable for institutions attempting to characterize the performance of such systems.

\section{Conflict of interest}

The authors declare that they have no conflicts of interest. The authors alone are responsible for the content and writing of the paper.

\section{References}

1. Mellenberg DE, Dahl RA, Blackwell CR. Acceptance testing of an automated scanning water phantom. Med Phys. 1990;17:311-4.

2. Purdy JA, American Association of Physicists in Medicine. Advances in Radiation Oncology Physics Dosimetry, Treatment Planning, and Brachtherapy: Medical Physics Monograph, No. 19, American Institute of Physics; New York, NY: 1992: 111-47.

3. Das IJ, Cheng CW, Watts RJ, et al. Accelerator beam data commissioning equipment and procedures: report of the TG-106 of the Therapy Physics Committee of the AAPM. Med Phys. 2008;35:4186-215.

4. Peng JL, Ashenafi MS, McDonald DG, Vanek KN. Assessment of a three-dimensional (3D) water scanning system for beam commissioning and measurements on a helical tomotherapy unit. J Appl Clin Med Phys. 2015;16:4980.

5. García-Vicente F, Béjar MJ, Pérez L, Torres JJ. Clinical impact of the detector size effect in 3D-CRT. Radiother Oncol. 2005;74:315-22.

6. Cranmer-Sargison G, Weston S, Sidhu NP, Thwaites DI. Experimental small field 6 MV output ratio analysis for various diode detector and accelerator combinations. Radioth Oncol. 2011;100:429-435.

7. Sharma SC, Ott JT, Williams JB, Dickow D. Commissioning and acceptance testing of a CyberKnife linear accelerator. J Appl Clin Med Phys. 2007;8:2473.

8. Patel B, Syh J, Durci M, et al. Comparison of TomoScanner ${ }^{\mathrm{TM}}$ 2D Water Phantom versus IBA Helix for Tomotherapy Profile Measurements. Med Phys. 2012;39:3734.

9. Akino Y, Gibbons JP, Neck DW, et al. Intra- and intervariability in beam data commissioning among water phantom scanning systems. J Appl Clin Med Phys. 2014;15:4850. 
10. Bakhtiari M. Effect of surface waves on radiotherapy dosimetric measurements in water tanks. J Med Phys. 2011;36:230-3.

11. Dieterich S, Ford E, Pavord D, Zeng J. Practical Radiation Oncology Physics: A Companion to
Gunderson Tepper's Clinical Radiation Oncology. Philadelphia, PA: Elsevier; 2015.

12. Mayles P, Nahum A, Rosenwald JC. Handbook of Radiotherapy Physics: Theory and Practice. Boca Raton, FL: Taylor \& Francis Group; 2007. 